Journal of Applied Fluid Mechanics, Vol. 9, Special Issue 1, pp. 113-120, 2016.

Selected papers from the $7^{\text {th }}$ International Exergy, Energy

and Environment Symposium, IEEE7-2015

Available online at www.jafmonline.net, ISSN 1735-3572, EISSN 1735-3645.

DOI: $10.36884 /$ jafm.9.SI1.25821

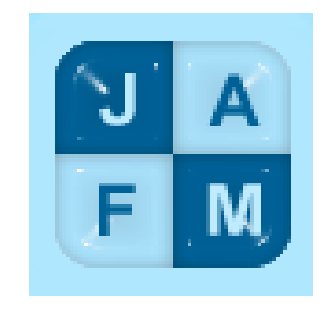

\title{
Experimental Study of Heat and Mass Transfer for Liquid Film Evaporation along a Vertical Plate Covered With a Porous Layer
}

\author{
A. Terzi ${ }^{12 \dagger}$, S. Ben Jabrallah ${ }^{12}$ and S. Harmand ${ }^{3}$ \\ ${ }^{1}$ Laboroitoire d'Energétique et des transferts thermique et massique, Faculté des sciences de Tunis, \\ Université Tunis El Manar, Tunisie \\ ${ }^{2}$ Faculté des sciences de Bizerte, 7021 Bizerte, Université de Carthage, Tunisie \\ ${ }^{3}$ Université de Lille Nord de France, F-59000 Lille UVHC, LAMIH CNRS UMR 8201, F-59313 \\ Valenciennes Cedex, France \\ †Corresponding Author Email: terziamine@hotmail.fr
}

(Received October 20, 2015; accepted December 10, 2015)

\begin{abstract}
In this paper, we realized an Experimental study of heat and mass transfer for liquid evaporation along a vertical plate covered with a porous layer. To develop this study, an experimental dispositive was realized. To highlight the effect of the addition of a porous layer on the phenomenon of evaporation, we first study the case of the flow of a liquid film on an aluminium plate. Then we covered the same plate by a porous layer. We could measure the temperature along the plate and the evaporated flow using the test bed. From these measurements we note that temperatures are higher with the presence of the porous medium which affect positively on the evaporated flow. In addition, various dimensionless numbers were analyzed as the sensible and latent local Nusselt number, solving the energy equation by inverse method. We note that the latent Nusselt number is more important than the sensible Nusselt Number. Then the flow dissipated by evaporation is greater than that used by the film to increase its temperature. We also note that the calculated values of the latent and sensible Nusselt number are greater in the presence of the porous medium that proves that the addition of the porous layer improves heat and mass exchange.
\end{abstract}

Keywords: Heat and mass transfer; Evaporation; Porous layer.

\section{NOMENCLATURE}

$\begin{array}{ll}A & \text { amplitude of oscillation } \\ a & \text { cylinder diameter } \\ \text { Cp } & \text { pressure coefficient } \\ \text { Cx } & \text { force coefficient in the } \mathrm{x} \text { direction } \\ \mathrm{Cy} & \text { force coefficient in the y direction } \\ \mathrm{C} & \text { chord } \\ \mathrm{dt} & \text { time step } \\ \mathrm{X} & \text { component of the resultant pressure force }\end{array}$

\section{INTRODUCTION}

The phenomena of heat and mass transfer are of considerable interest in the engineering field. This interest is reflected in many applications such as desalination, distillation, drying and cooling of electronic components.

Indeed, this topic has been the subject of several research studies for many years and brought together several lines of scientific work.

To improve the transfer, researchers have studied

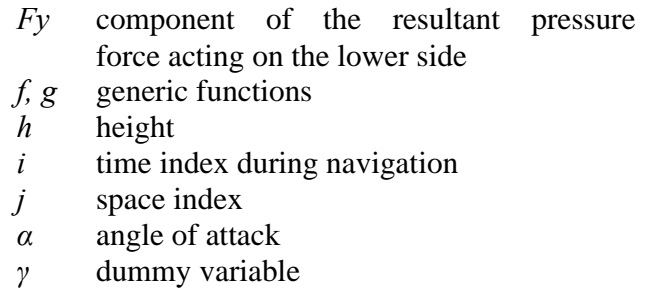

different geometries and conducted a parametric study on almost all input parameters that may influence transfers.

For instance, there are those who have studied the evaporation of a liquid film flowing on a flat plate (Siow, 2002).

Cherif, (2011) addresses the case of the evaporation of a liquid film on a vertical plate.

There are other researchers who are interested in the study of evaporation on an inclined plane, which 


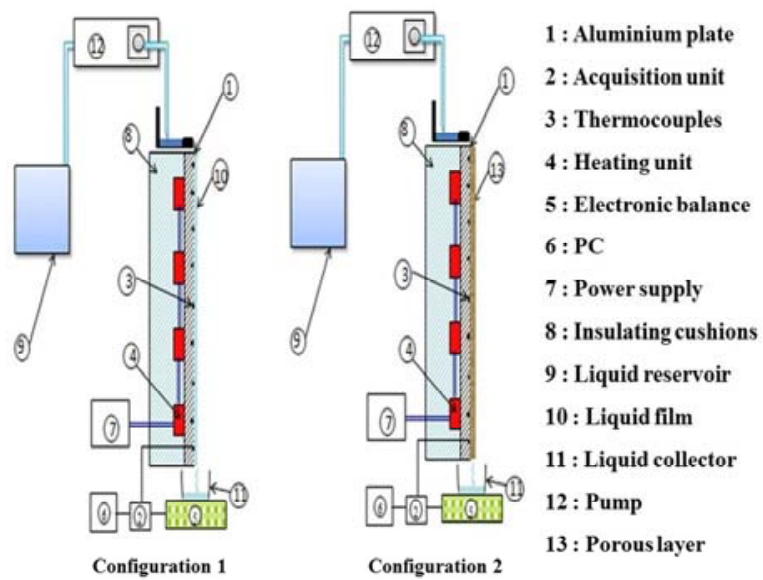

Fig. 1. The studied configurations.

affects gravitational forces and decreases the rate of fluid flow (Agunaoun, 1994).

In the other hand, the use of binary fluids was another solution to improve the heat and mass exchange (Debbissi, 2013).

However, obtaining a homogenous liquid film over the entire plate constitutes a major discrepancy between the theoretical and experimental studies. Despite efforts in the field of modeling and numerical simulation, we still see a difference between calculation and experiment. In a previous work of Cherif (2011), they have studied the two aspects of evaporation film: experiment and simulation. A difference was reported. They believe that this difference is caused by the difficulty of making a falling film on a vertical plate. In fact, the film can't be controlled if it is directly adhered to the plate.

To analyze the effect of dry zones on the plate, Mammou (1992) numerically studied the evaporation along an inclined plate. This plate consists of two wet zones separated by dry zone. The results of this study showed that the length of the dry zone plays an important role.

More recent studies have explored various techniques to solve this problem. For example, several researchers have used rough surfaces, interposed obstacles (Zheng 1999), used a porous layer that covers the plate or have used a corrugated plate (Gonda, 2014).

As a result, this work focuses on the study of the flow of a liquid film on a vertical plane covered by a porous layer. The main objective is to evaluate the effect of the presence of the porous layer on the phenomenon of evaporation.

\section{EXPERIMENTAL FACILITY}

The following section outlines general (nonformatting) guidelines to follow. These guidelines are applicable to all authors and include information on the policies and practices relevant to the publication of your manuscript.

\subsection{Setup}

To conduct the study, we realized an experimental setup, completed by a measurement system that allows the automatic acquisition of the temperatures measured by the different thermocouples installed along the plate.

The experimental setup is composed of a plate of aluminum in size $1000 * 500 * 12 \mathrm{~mm}$. Electrical resistors connected to the generator are distributed homogeneously over the entire rear face of the plate which forms the heating system. To avoid losses of heat flow, a glass wool layer and a Plexiglas wall were placed around resistances. We set a reservoir of water above the plate, which, once full, it is drained by overflow, thus creating water film flowing homogeneously on the exchange surface.

After the realization of all tests for the evaporation of a liquid film on a vertical plate (configuration 1), we covered the exchange surface with a porous layer and thus, we passed to the second configuration.

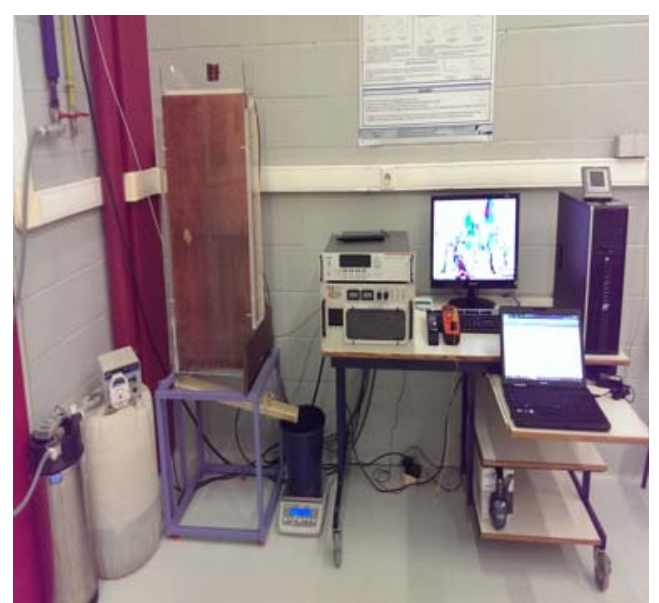

Fig. 2. Schematic of the experimental setup. 


\subsection{Measurements}

To study the effect of the presence of the porous medium on the evaporation phenomenon, we first conducted the experimental tests for the case of the flow of a liquid film on a vertical plate (configuration 1). Next, we spent the second configuration by covering the plate with a porous layer of the same size and thickness of $1 \mathrm{~cm}$.

The heat flow and the water inlet flow appear as operating parameters whose influence should be studied. For this, tests are realized for different heating power from $400 \mathrm{~W}$ to $1400 \mathrm{~W}$ and for two water inlet flow $(\mathrm{min}=2.77 \mathrm{~g} \cdot \mathrm{m}-2 . \mathrm{s}-1$ and $\mathrm{min}=$ 4.44 g.m-2.s-1)

Table 1 contains the variation ranges of the experimental conditions.

\section{RESULTS AND DISCUSSIONS}

\subsection{Temperature evolutions}

Before starting the tests in the presence of the liquid film, it is interesting to study the effect of adding the porous layer in natural convection without phase

Table 1 Variation range of the experimental conditions

\begin{tabular}{|c|c|c|}
\hline \multicolumn{3}{|c|}{ conditions } \\
\hline Variables & Minimum & Maximum \\
\hline $\begin{array}{c}\text { Ambient } \\
\text { temperature }\left({ }^{\circ} \mathrm{C}\right)\end{array}$ & 16 & 25 \\
\hline $\begin{array}{c}\text { Inlet water } \\
\text { temperature }\left({ }^{\circ} \mathrm{C}\right)\end{array}$ & 15 & 22 \\
\hline $\begin{array}{c}\text { Water inlet flow } \\
\left(\mathrm{g} \cdot \mathrm{m}^{-2} . \mathrm{s}^{-1}\right)\end{array}$ & 2.77 & 4.44 \\
\hline Heat flow $\left(\mathrm{W} \cdot \mathrm{m}^{-2}\right)$ & 800 & 2800 \\
\hline Humidity (\%) & 40 & 65 \\
\hline
\end{tabular}

change (without liquid). For this, we represent in fig 3 the temperature variation throughout the plate without and with porous medium.

For reasons of safety and compliance with operating limits of heated components, we have chosen to study the case of natural convection only for a heat flow equal to 800 W.m-2. First, we note that the

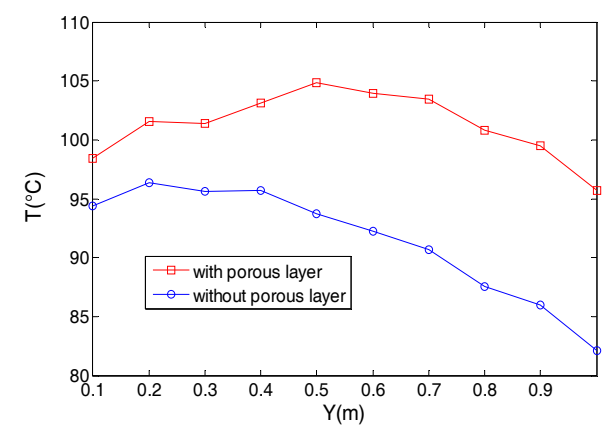

Fig. 3. Variation of the temperature throughout the plate in natural convection $\left(q=800 \mathrm{~W} \cdot \mathrm{m}^{-2}\right)$. temperature increases from bottom to top of the plate, the area near the plate is hotter, the density decrease and therefore creating a flow of air near of the plate. Fresh air is sucked towards the plate which explains this temperature difference between the upper area and the lower area of the plate. Moreover, we note that the measured temperatures are much higher in the presence of the porous medium. We will see later, the effect of the increase of temperature on the evaporation phenomena.

We represent in Fig. 4, the evolution of the temperature along the plate for different heat flow and different liquid inlet flow. As in the case of natural convection, the temperature measured in the presence of the porous layer are higher. Indeed, the fact of covering the plate by a porous layer creates a kind of thermal resistance at the exchange area, which will be beneficial for the evaporation process. Moreover, we note that the profiles of the temperature are divided into two areas: the first area begins from the top of the plate (entrance of liquid) to the point where the temperature reaches its maximum. In this area of the plate, the liquid heats up during its descent without significant evaporation, this explains the linear and remarkable increase of the temperature. From the point where the temperature reaches its maximum to the bottom of the plate, we note that the temperature is almost constant: the evaporation zone.

This result is proved in the work of Jabrallah (2005), who has demonstrated the existence of these two zones in the case of the evaporation of a film liquid flowing on the wall of a vertical cavity.

It is noted also that during the evaporation without the porous medium, the heating zone defined previously, is spread over a larger area. Thus the liquid is heated more quickly and the temperature becomes more stable in the presence of the porous medium.

Obviously, the temperature increase with increasing the heat flow. However, we find that in the presence of the porous layer, the temperature measured for a heat flow equal to $2400 \mathrm{~W} . \mathrm{m}-2$ and $2800 \mathrm{~W} . \mathrm{m}-2$ are almost identical. So it can be concluded that the addition of energy brought to the liquid is completely consumed by the phase change, which proves that the presence of the porous medium minimizes the loss of energy by convection.

\subsection{Resolution of the heat equation by the inverse method}

Certainly, knowing the temperature at any point of the wet wall requires special interest in understanding the phenomenon of evaporation. But the knowledge of the local variation of the evaporated flow is essential. For this, and from the values of the measured temperatures, we must solve the heat equation on the plate. This resolution allows us to determine the values of the exchange coefficient at the wet wall and determine subsequently the variation of evaporated flow. The heat equation can be expressed by: 


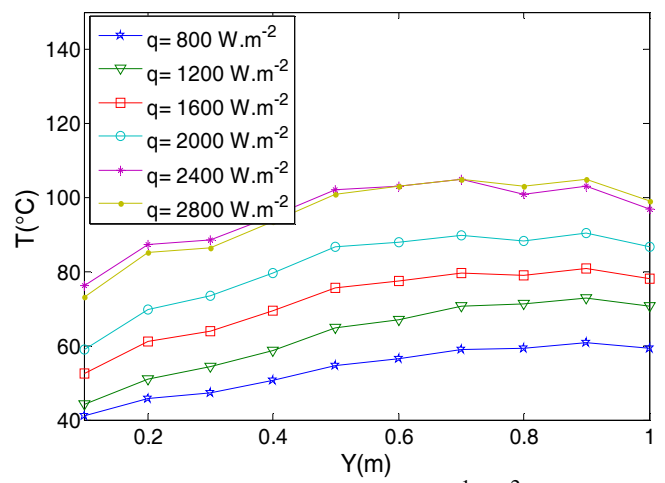

A-1: $m_{\text {in }}=2.77$ g.s s $^{-1} \cdot \mathrm{m}^{-2}$

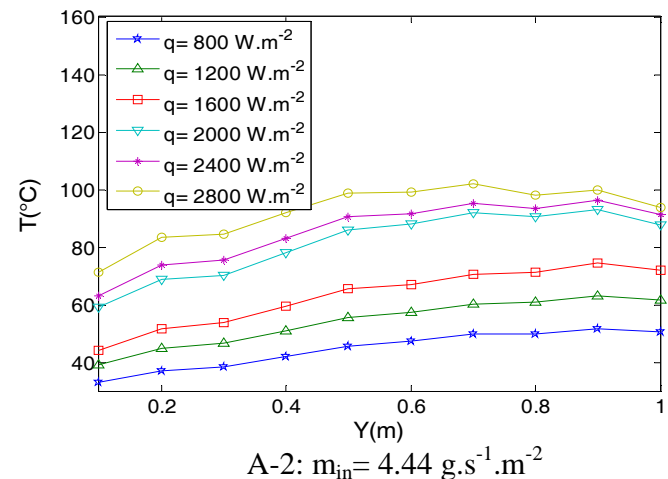

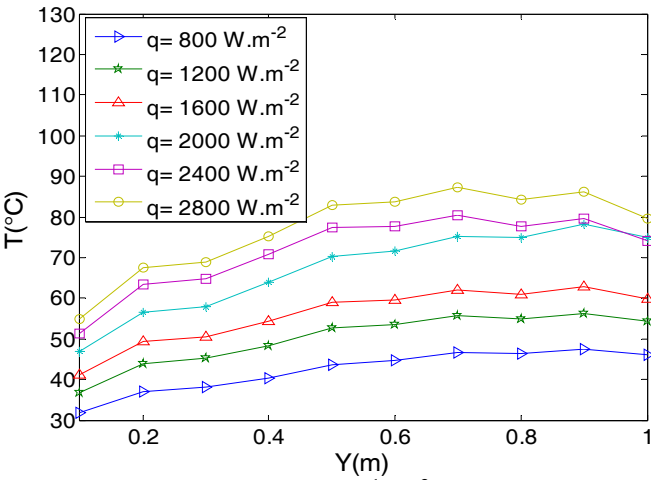

B-1: $\mathrm{m}_{\text {in }}=2.77 \mathrm{~g} \cdot \mathrm{s}^{-1} \cdot \mathrm{m}^{-2}$

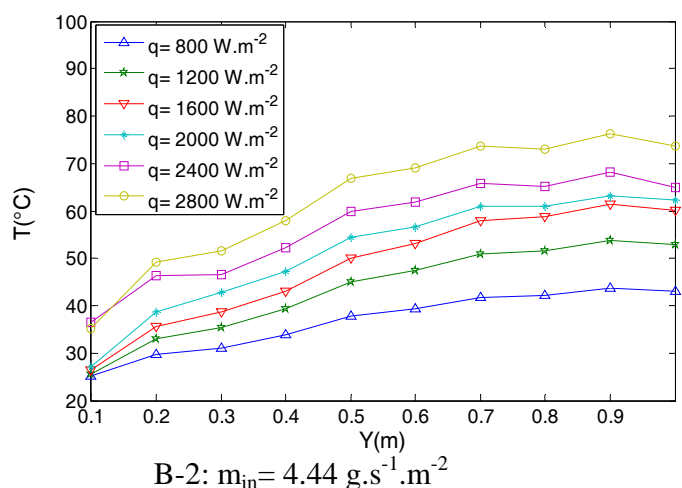

Fig. 4. Variation of the temperature throughout the plate for different heat flow and different liquid inlet flow (A: With porous layer and B: Without porous layer).

$$
\begin{aligned}
& p(x, y)+\lambda\left(\beta \frac{\partial^{2} T}{\partial x^{2}}+\gamma \frac{\partial^{2} T}{\partial y^{2}}\right)= \\
& \frac{1}{e}\left(\phi_{a r}+\phi_{a v}\right)+\alpha \rho C_{p} \frac{\partial T}{\partial t}
\end{aligned}
$$

Where, $\mathrm{p}$ is the intern production (W.m-3), assumed to be homogeneous throughout the electrical resistance. , is the flux density dissipated by the back (insulator) and the desired flux density corresponding to convection and radiation on the upper side (air-cooled) of the resistor.

To simplify the problem, we will assume that the problem is stationary and in two dimensions, which means that:

$\frac{\partial T}{\partial t}=0$

So the equation takes the following form:

$$
p(x, y)+\left(\frac{\partial^{2} T}{\partial x^{2}}+\frac{\partial^{2} T}{\partial y^{2}}\right)=\frac{1}{e}\left(\phi_{a v}\right)
$$

The problem is a priori well-posed, since it has only one unknown, which is the conductive flux, and could therefore be the subject of a direct resolution. But the problem is the precision of the determination of the conductive flow in the resistance from the experimentally measured temperatures, which are noisy. It is more correct to implement an inverse resolution for this problem. More precisely the flow is determined by solving an inverse conduction problem, coupled to the direct thermal modeling of the general equation shown above, by finite differences. The principle is to impose the desired flow in the direct model and retrieve temperatures so deducted. The inverse method consists in the correction of the flow imposed from the comparison between the measured and numerical temperatures. So we must turn the difference between the temperatures calculated by direct method and measured temperatures to 0 . Thus we aim to minimize the following criterion:

$$
F=\sum_{x} \sum_{y}\left(T_{c a l}(x, y)-T_{m e s}(x, y)\right)^{2}
$$

The minimization of this criterion alone does not regulate research and gives free rein to the amplification of errors in input data. Thus, we follow the method described by (Beck 1996 and Petit 2008). It consists of 

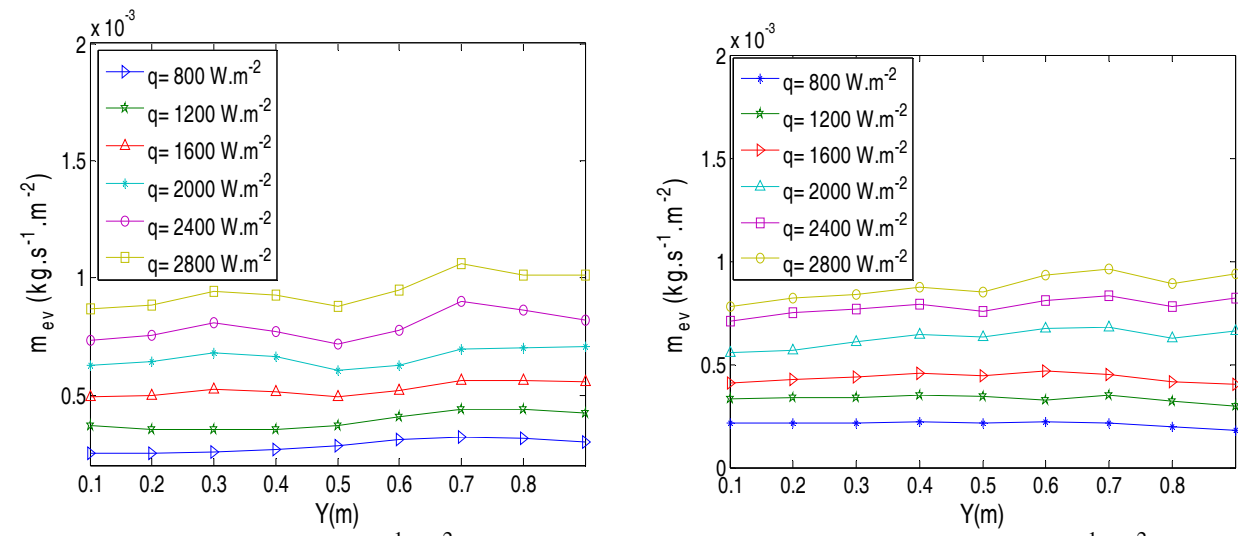

A-1: $\mathrm{m}_{\mathrm{in}}=2.77$ g.s $\mathrm{s}^{-1} \cdot \mathrm{m}^{-2}$

B-1: $\min =2.77 \mathrm{~g} \cdot \mathrm{s}^{-1} \cdot \mathrm{m}^{-2}$
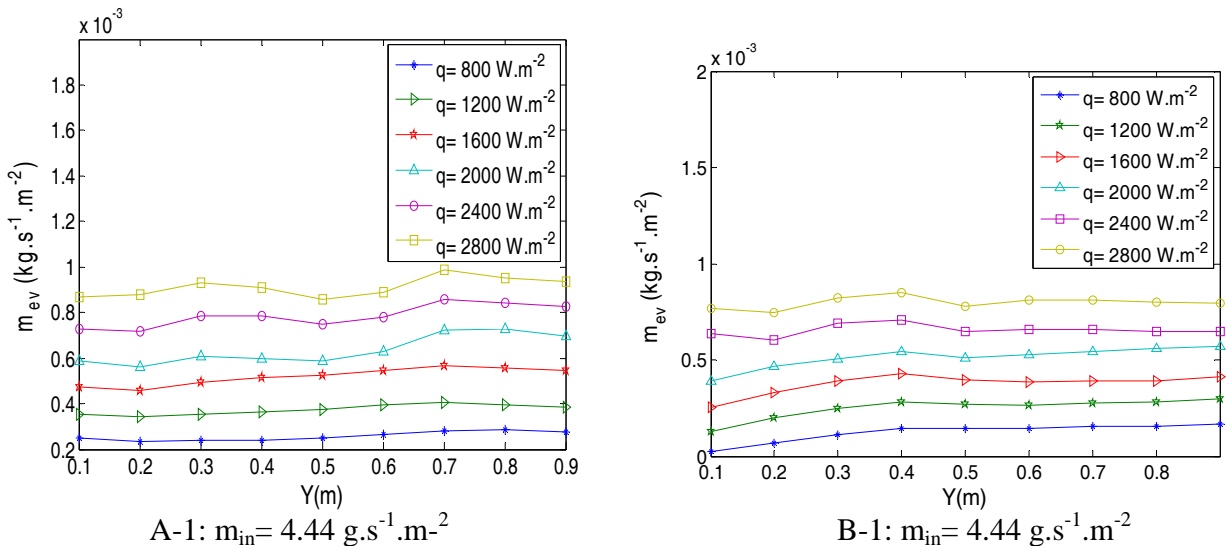

Fig. 5. Local variation of the evaporated flow throughout the plate for different heat flows and different liquid inlet flow (A: With porous layer and B: Without porous layer).

adding the regularization terms to the previous criterion, and therefore we get:

$$
\begin{aligned}
& F=\sum_{x} \sum_{y}\left(T_{c a l}(x, y)-T_{m e s}(x, y)\right)^{2} \\
& +\alpha_{1} \sum_{x} \sum_{y}\left(\operatorname{grad}\left(\phi_{a v}(x, y)\right)\right)^{2}
\end{aligned}
$$

\subsection{Evaporated flow}

Fig 5 shows the local variation of the evaporated flow along the plate for different heat flow and different liquid inlet flow.

The heat flow is the essential source of energy needed to the liquid-gas change phase. By observing the variation of the evaporated flow, we find that an increase in the heat flow leads to an improved evaporated flow. Indeed, an increase in the heating density expresses an energy addition which results in increases in thermal and mass gradients.

On the other hand, we see that increasing the liquid inlet flow, decrease the evaporated flow. This effect is explained by the fact that the contact time

between the film and the heated plate is shorter when the inlet flow is important. Indeed the film flows faster on the plate. So we can conclude that to upgrade evaporation, the system must operate at low water inlet flow.

Moreover, we note that the evaporated quantities in the presence of the porous medium are more important. This is due to two major effects. The first, as mentioned previously, the temperatures measured in the presence of the porous layer are higher. The second effect is that the fact to cover the plate by a porous layer, improves the wettability. Indeed, the liquid infiltrates in the pores and thus covers the totality of the exchange surface.

\section{Nusselt number}

In order to generalize our study, heat and mass transfer along the plate were described by dimensionless numbers. The transfer of heat exchanged at the interface between the film of water and air is the sum of the convective flux and latent flux (Fedorov 1997).

$$
\phi_{T}=\phi_{S}+\phi_{L}
$$

The local Nusselt number at the interface is defined as: 


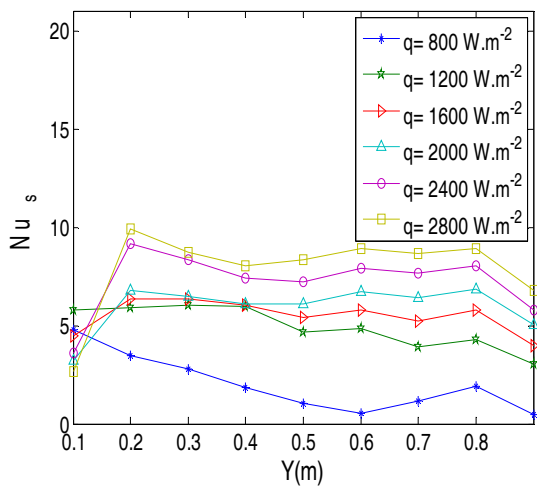

A-1: $\mathrm{m}_{\mathrm{in}}=2.77 \mathrm{~g} \cdot \mathrm{s}^{-1} \cdot \mathrm{m}^{-2}$

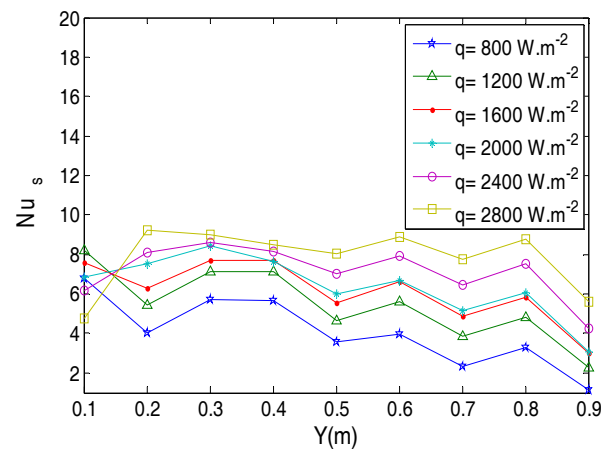

A-2: $\mathrm{m}_{\mathrm{in}}=4.44 \mathrm{~g} \cdot \mathrm{s}^{-1} \cdot \mathrm{m}^{-2}$

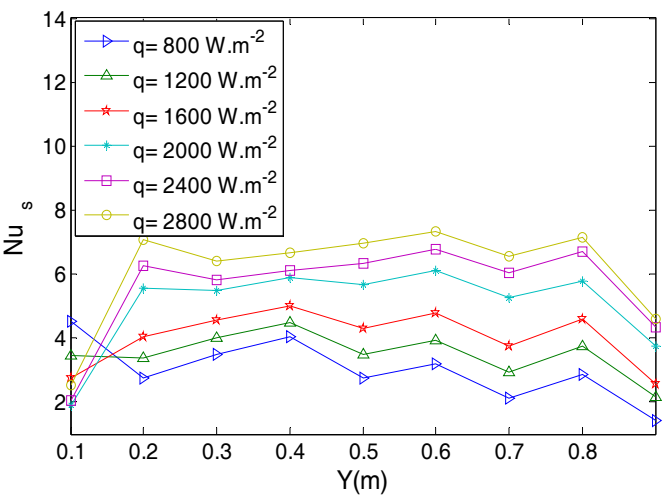

B-1: $\mathrm{m}_{\mathrm{in}}=2.77 \mathrm{~g} \cdot \mathrm{s}^{-1} \cdot \mathrm{m}^{-2}$

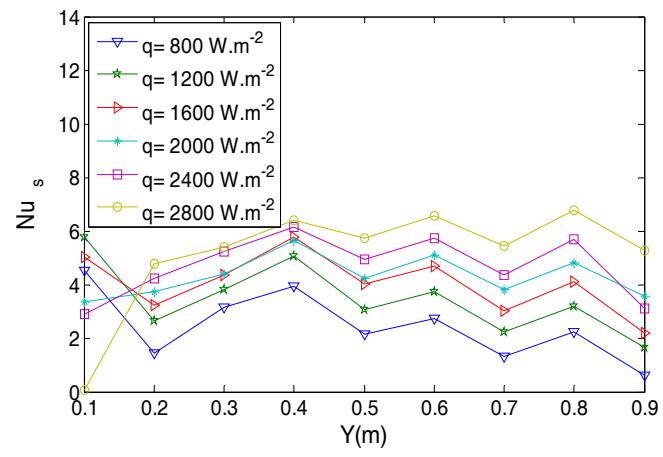

B-2: $\mathrm{m}_{\text {in }}=4.44 \mathrm{~g} \cdot \mathrm{s}^{-1} \cdot \mathrm{m}^{-2}$

Fig. 6. Variation of the local sensible Nusselt number throughout the plate for different heat flows and different liquid inlet flow (A: With porous layer and B: Without porous layer)

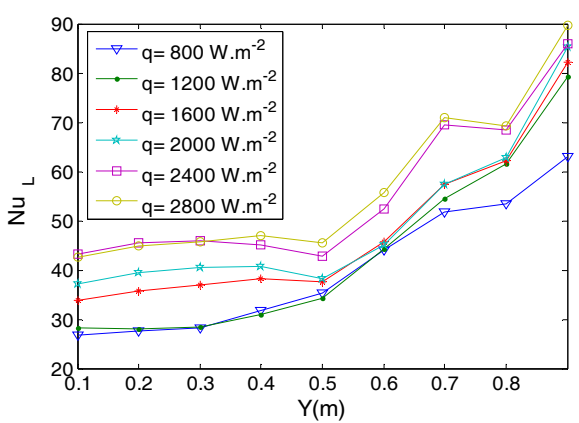

A-1: $m_{\text {in }}=2.77$ g.s s $^{-1} \cdot \mathrm{m}^{-2}$

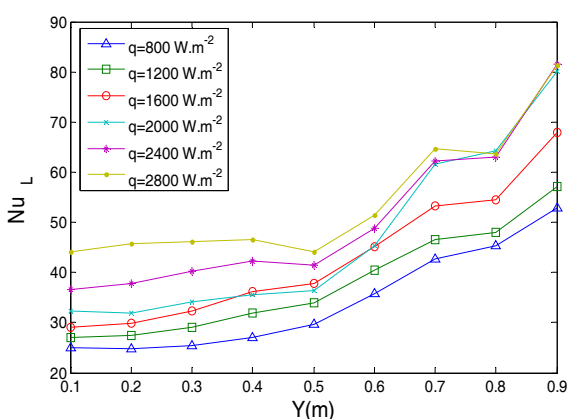

A-1: $\mathrm{m}_{\mathrm{in}}=4.44 \mathrm{~g} \cdot \mathrm{s}^{-1} \cdot \mathrm{m}^{-2}$

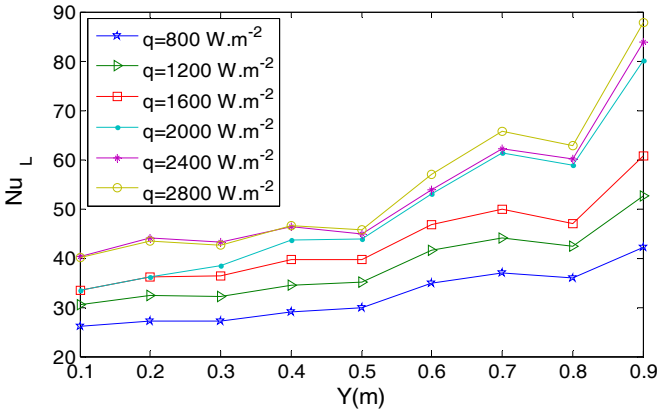

B-1: $\mathrm{m}_{\mathrm{in}}=4.44 \mathrm{~g} \cdot \mathrm{s}^{-1} \cdot \mathrm{m}^{-2}$

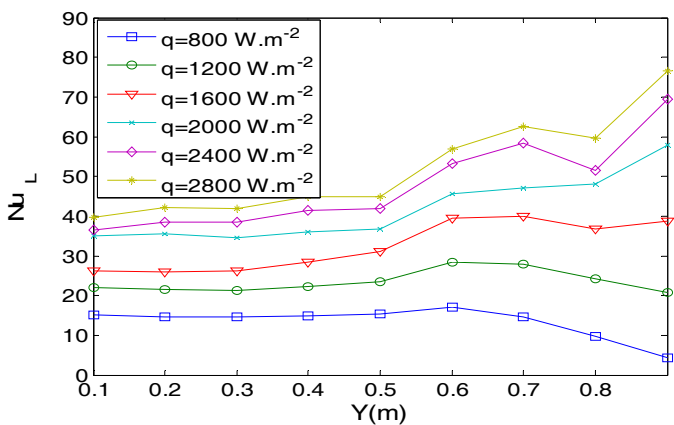

B-1: $\mathrm{m}_{\text {in }}=4.44 \mathrm{~g} \cdot \mathrm{s}^{-1} \cdot \mathrm{m}^{-2}$

Fig. 7. Variation of the local latent Nusselt number throughout the plate for different heat flows and different liquid inlet flow (A: With porous layer and B: Without porous layer) 


$$
N u=\frac{\phi_{T}}{\phi_{c d}}
$$

May be consisted of two dimensionless numbers:

$N u=N u_{S}+N u_{L}$

Where, Nus and NuL represent the sensible local Nusselt number and the latent local Nusselt number, describing respectively the sensible and latent transfer:

$$
\begin{aligned}
& N u_{S}=\frac{\varphi_{S} \cdot L}{\lambda \cdot\left(T_{p}-T_{L_{\text {in }}}\right)} \\
& N u_{L}=\frac{\varphi_{L} \cdot L}{\lambda \cdot\left(T_{p}-T_{L_{\text {in }}}\right)}
\end{aligned}
$$

Fig. 6 represent the variation of the sensible local Nusselt number throughout the plate, for different heat flow and different liquid inlet flow.

As the evaporated flow, we note that the sensible Nusselt number increases with the increase of the heat flow and the decrease of the liquid inlet flow. We also note that the calculated values of the Nusselt number are significantly greater in the presence of the porous medium. Indeed, the use of a porous layer of copper improves the transfer from the heated plate to the liquid- gas interface, due to its high conductivity, which promotes the phase change and can evacuate more energy.

We represent in Fig 7 the variation of the latent local Nusselt number along the plate for different heat flux and different liquid inlet flows. First, we note that the latent Nusselt number increases with increasing heat flow and decreasing the liquid inlet flow.

We also note that the latent Nusselt number is more important than the sensible Nusselt number. The flow dissipated by evaporation is greater than that used by the film to increase its temperature.

In addition, the calculated values of the latent Nusselt number are greater in the presence of the porous medium that proves that the addition of the porous layer improves the mass exchange and therefore the evaporated flow.

\section{Conclusions}

This work focuses on the study of the flow of a liquid film on a vertical plane covered by a porous layer. The main objective is to evaluate the effect of the presence of the porous layer on the phenomenon of evaporation.

The most significant conclusions are as follows:

1-The liquid is heated more quickly and the temperature becomes more stable in the presence of the porous medium.

2-In the presence of the porous layer, the temperature measured for a heat flow equal to 2400 W.m-2 and 2800 W.m-2 are almost identical. So it can be concluded that the addition of energy brought to the liquid is completely consumed by the phase change, which proves that the presence of the porous medium minimizes the loss of energy by convection.

3-The evaporated quantities in the presence of the porous medium are more important. This is due to two major effects: The first is that the temperatures measured in the presence of the porous layer are higher. The second effect is that the fact to cover the plate by a porous layer, improves the wettability.

4-The Nusselt number are significantly greater in the presence of the porous medium. Indeed, the use of a porous layer of copper improves the transfer from the heated plate to the liquid- gas interface, due to its high conductivity, which promotes the phase change and can evacuate more energy.

5-the latent Nusselt number are greater in the presence of the porous medium that proves that the addition of the porous layer improves the mass exchange and therefore the evaporated flow.

6-The latent Nusselt number is more important than the sensible Nusselt number. The flow dissipated by evaporation is greater than that used by the film to increase its temperature.

For unpublished lectures of symposia, include title of paper, name of sponsoring society in full, and date. Give titles of unpublished reports with "(unpublished)" following the reference. Only articles that have been published or are in press should be included in the references. Unpublished results or personal communications should be cited as such in the text.

\subsection{Books and Theses}

The title of books and theses are appeared in italic format followed by the type of thesis (e.g. MSc thesis), the University or publisher name, city, and country.

\subsection{Journals}

For journals, only the name of journal appears in italic format followed by non-italic volume (number) and the paper page numbers; e.g. International Journal of Analytical and Experimental Modal Analysis 7(2), 111-127. The journal issue number (2) is preferred, but the month (Nov.) can be substituted if the issue number is not available. Use the complete date for daily and weekly publications. Transactions follow the same style as other journals; if punctuation is necessary, use a colon to separate the transactions title from the journal title.

\subsection{Proceedings}

For conference proceedings, only the name of 
conference appears in italic format followed by non-italic city and country and the paper page numbers.

\subsection{Web pages}

For periodicals all of the preceding information is required. The journal issue number ("No. 11" in Ref. 1) is preferred, but the month (Nov.) can be substituted if the issue number is not available. Use the complete date for daily and weekly publications. Transactions follow the same style as other journals; if punctuation is necessary, use a colon to separate the transactions title from the journal title.

Electronic publications, CD-ROM publications and regularly issued, dated electronic journals are permitted as references. Archived data sets also may be referenced as long as the material is openly accessible and the repository is committed to archiving the data indefinitely. References to electronic data available only from personal Web sites or commercial, academic, or government ones where there is no commitment to archiving the data are not permitted in the reference list.

The references should be grouped at the end of the paper in the alphabetical order of the last name of the first author in the style below.

\section{CONCLUSION}

Although a conclusion may review the main points of the paper, it must not replicate the abstract. A conclusion might elaborate on the importance of the work or suggest applications and extensions. Do not cite references in the conclusion as all points should have been made in the body of the paper. Note that the conclusion section is the last section of the paper to be numbered. The appendix (if present), acknowledgment, and references are listed without numbers.

\section{ACKNOWLEDGEMENTS}

The Chief Editor of JAFM would like to thank all authors for their contributions and the submission of their papers.

\section{REFERENCES}

Agunaoun, A. and A. Daif (1994). Evaporation en convection forcée d'un film mince s'écoulant en régime permanent, laminaire et sans onde, sur une surface plane inclinée. International Journal of Heat and Mass transfer (18), 29472956.
Beck, J. V, B. Blackwell, and A. Haji-Sheikh (1996). Comparison of some inverse heat conduction methods using experimental data, International Journal Heat and Mass Transfer (39), 3649-3657.

Ben, Jabrallah, S. A. Belghith, and J. P. Corriou (2005). Convective heat and mass transfer with evaporation of a falling film in a cavity. International Journal of Thermal Sciences (45) 16-28.

Cherif, A. S, S. Ben Jabrallah, J. P. Corriou and A. Belghith (2010). Intensification of the liquid film evaporation in a vertical channel. Desalination (250), 433-437.

Cherif, A. S, M. A. Kassim, B. Benhamou, S. Harmand, J. P. Corriou, and S. Ben Jabrallah (2011). Experimental and numerical study of mixed convection heat and mass transfer in a vertical channel with film evaporation. International Journal of Thermal Sciences (50), 942-953.

Fedorov, A. G, R. Viskanta, and A. Mohamad (1997). Turbulent heat and mass transfer in asymmetrically heated, vertical parallel plate channel. International Journal Heat Fluid Flow (18) 308-315.

Gonda, A. P. Lancereau, P. Bondelier, L. Luo, Y. Fan, S. Benezch (2014). Water falling film evaporation on a corrugated plate. International Journal of Thermal Sciences (81) 29-37

Mammou, M., M. Daguenet, and G. Le Palec (1992). Numerical study of heat and mass transfer from an inclined flat plate with wet and dry zones. International Journal of Heat and Mass transfer (35) 2277-2287.

Petit, D. and D. Maillet (2008). Techniques inverses et estimation de paramètres. Techniques de l'ingénieur (AF4516), 1-24.

Siow, E. C, S. J. Ormiston, and H. M. Soliman (2002). Fully coupled solution of a two-phase model for laminar film condensation of vapor gas mixtures in horizontal channels. International Journal of Heat and Mass Transfer (45), 3689-3702.

Zheng, G. S, and W. M. Worek (1999). Method of heat and mass transfer enhancement in film evaporation. International Journal of Heat and Mass transfer (42) 861-872. 